\title{
Stability of the index of a linear relation under compact perturbations
}

\author{
by \\ Dana Gheorghe (Pitesti and Lille)
}

\begin{abstract}
We prove the stability under compact perturbations of the algebraic index of a Fredholm linear relation with closed range acting between normed spaces. Our main tool is a result concerning the stability of the index of a complex of Banach spaces under compact perturbations.
\end{abstract}

1. Introduction. The purpose of this paper is to show that the algebraic index of a Fredholm linear relation with closed range acting between normed spaces is stable under compact perturbations.

Let $X$ and $Y$ be two normed spaces. We denote by $\mathcal{B}(X, Y)$ the normed space of bounded linear operators from $X$ into $Y$ and by $\mathcal{K}(X, Y)$ the normed space of compact operators from $X$ into $Y$. A linear relation is a map $T$ from $X$ into $\mathcal{P}(Y):=\{A \subset Y: A \neq \emptyset\}$ such that

$$
T x+T y=T(x+y), \quad \alpha T x=T(\alpha x), \quad \forall x, y \in X, \forall \alpha \in \mathbb{C} \backslash\{0\} .
$$

We denote by $\operatorname{LR}(X, Y)$ the set of all linear relations from $X$ into $Y$. The kernel of $T \in \operatorname{LR}(X, Y)$ is the set $N(T)=\{x \in X: 0 \in T x\}$ and the range of $T$ is the set $R(T)=\bigcup_{x \in X} T x$. The linear relation $T$ is called Fredholm if $\operatorname{dim} N(T)<\infty$ and codim $R(T)<\infty$. In this case, the integer

$$
\operatorname{ind}(T):=\operatorname{dim} N(T)-\operatorname{codim} R(T)
$$

is called, as usual, the index of $T$. The main result of this article is the following.

TheOREM 1. Let $T \in \mathrm{LR}(X, Y)$ be a Fredholm linear relation with closed range. If $K \in \mathcal{K}(X, Y)$ is such that the range of $T+K$ is closed, then $T+K$ is Fredholm and $\operatorname{ind}(T)=\operatorname{ind}(T+K)$.

Note that if $T$ is a continuous Fredholm operator between Banach spaces, then $R(T)$ and $R(T+K)$ are closed linear subspaces of $Y$. In our case, the

2000 Mathematics Subject Classification: Primary 47A53; Secondary 47A06.

Key words and phrases: Fredholm operators, index, Banach space complexes, linear relations. 
condition " $R(T+K)$ is closed" becomes a hypothesis because of the possible lack of continuity of $T$.

Our main tool is a result of Ambrozie [1, Theorem 5] concerning the stability of the index of a complex of Banach spaces under compact perturbations. We state a particular case of this theorem in Section 2. To relate the index of a linear relation between normed spaces to the index of a complex of normed spaces we use an idea of $\mathrm{H}$. Zhang [8]. A similar result for the stability of the algebraic index of a linear relation under small perturbations has been proved in [4].

Acknowledgements. The author is grateful to Professor F.-H. Vasilescu for his constant help and encouragement.

2. Notation and preliminaries. Let $X_{i}(i=1,2,3)$ be normed spaces, and $\alpha_{i} \in \mathcal{B}\left(X_{i}, X_{i+1}\right)(i=1,2)$. The sequence

$$
0 \rightarrow X_{1} \stackrel{\alpha_{1}}{\longrightarrow} X_{2} \stackrel{\alpha_{2}}{\longrightarrow} X_{3} \rightarrow 0
$$

is called a complex if $R\left(\alpha_{1}\right) \subset N\left(\alpha_{2}\right)$. The complex (1) is said to be Fredholm if $\operatorname{dim} N\left(\alpha_{1}\right), \operatorname{dim} N\left(\alpha_{2}\right) / R\left(\alpha_{1}\right)$ and $\operatorname{codim} R\left(\alpha_{2}\right)$ are finite. Define the index of (1) by

$$
\operatorname{ind}(1):=\operatorname{dim} N\left(\alpha_{1}\right)-\operatorname{dim} N\left(\alpha_{2}\right) / R\left(\alpha_{1}\right)+\operatorname{codim} R\left(\alpha_{2}\right) .
$$

The next result is a particular case of Theorem 5 in [1] (see also [2]), and will be used to prove our main result.

Theorem 2. Let $X_{i}(i=1,2,3)$ be Banach spaces. Assume that the complex (1) is Fredholm and $\beta_{i} \in \mathcal{B}\left(X_{i}, X_{i+1}\right)(i=1,2)$ are such that the sequence

$$
0 \rightarrow X_{1} \stackrel{\beta_{1}}{\longrightarrow} X_{2} \stackrel{\beta_{2}}{\longrightarrow} X_{3} \rightarrow 0
$$

is a complex. If $\beta_{i}-\alpha_{i} \in \mathcal{K}\left(X_{i}, X_{i+1}\right)(i=1,2)$, then (3) is Fredholm and

$$
\operatorname{ind}(3)=\operatorname{ind}(1) \text {. }
$$

Remark 1. The above theorem is due to A. S. Faĭnshteĭn and V. S. Shul'man. On the other hand, to prove our main result we may also use a result of F.-H. Vasilescu [7] where $\alpha_{i}, \beta_{i}(i=1,2)$ are as above and satisfy the supplementary condition that $\left(\beta_{2}-\alpha_{2}\right)\left(\beta_{1}-\alpha_{1}\right)$ is of finite rank.

Let $X$ and $Y$ be normed spaces, $X_{0} \subset X$ a closed linear subspace and $Y_{0} \subset Y$ a linear subspace. Let $T_{0}: X \rightarrow Y$ be a linear operator such that $T_{0}\left(X_{0}\right) \subset Y_{0}$. Then $T_{0}$ induces the linear transformation $T: X / X_{0} \rightarrow Y / Y_{0}$ defined by

$$
T\left(x+X_{0}\right)=T_{0} x+Y_{0}, \quad \forall x \in X .
$$

Let $N(T)$ be the kernel of $T, R(T)$ the range of $T, G(T)$ the graph of $T, G_{0}(T):=\left\{(x, y) \in X \times Y: T\left(x+X_{0}\right)=y+Y_{0}\right\}$ and $R_{0}(T):=$ 
$\left\{y \in Y: y+Y_{0} \in R(T)\right\}$. Notice that $R(T)=R_{0}(T) / Y_{0}, G_{0}(T)=$ $G\left(T_{0}\right)+X_{0} \times Y_{0}$ and $R_{0}(T)=R\left(T_{0}\right)+Y_{0}$. The transformation $T$ is said to be Fredholm if $\operatorname{dim} N(T)$ and $\operatorname{codim} R(T):=\operatorname{dim}\left(Y / Y_{0}\right) / R(T)$ are finite, and we define

$$
\operatorname{ind}(T)=\operatorname{dim} N(T)-\operatorname{codim} R(T)
$$

to be the index of $T$.

We associate to $T$ the sequence of normed spaces

$$
0 \rightarrow X_{0} \stackrel{i_{T}}{\longrightarrow} X \times Y_{0} \stackrel{j_{T}}{\longrightarrow} Y \rightarrow 0,
$$

where

$$
\begin{aligned}
i_{T}\left(x_{0}\right) & =\left(x_{0}, T_{0} x_{0}\right), \quad \forall x_{0} \in X_{0}, \\
j_{T}\left(x, y_{0}\right) & =T_{0} x-y_{0}, \quad \forall x \in X, y_{0} \in Y_{0} .
\end{aligned}
$$

It is easy to see that (4) is a complex.

LEmma 1. The linear transformation $T$ is Fredholm iff the complex (4) is Fredholm, and in this case,

$$
\operatorname{ind}(T)=-\operatorname{ind}(4) .
$$

Proof. Define

$$
\alpha: N\left(j_{T}\right) \rightarrow X / X_{0}, \quad \alpha\left(\left(x, T_{0} x\right)\right)=x+X_{0} .
$$

Clearly $R(\alpha)=N(T)$ and $N(\alpha)=R\left(i_{T}\right)$, thus $N\left(j_{T}\right) / R\left(i_{T}\right)$ is algebraically isomorphic to $N(T)$. Define

$$
\beta: Y \rightarrow Y / R_{0}(T), \quad \beta(y)=y+R_{0}(T) .
$$

The map $\beta$ is surjective and $N(\beta)=R\left(j_{T}\right)$, thus $Y / R\left(j_{T}\right)$ is algebraically isomorphic to $Y / R_{0}(T) \cong\left(Y / Y_{0}\right) / R(T)$. The proof is complete.

Lemma 2. Let $X, Y$ be normed spaces, $M \subset X$ a linear subspace of $X$, $A \subset X$ an arbitrary subset of $X, T \in \mathcal{B}(X, Y)$ and $T^{\prime} \in \mathcal{B}\left(Y^{\prime}, X^{\prime}\right)$ the adjoint of $T$. Let $B_{X}$ and $B_{Y}$ be the closed unit balls in $X$ and $Y$, respectively. Then

(i) $\quad N\left(T^{\prime}\right)=R(T)^{\perp}$ and $N(T)=R\left(T^{\prime}\right)^{\perp}$ (where $\perp$ denotes the annihilator or preannihilator of a set).

(ii) $\left(B_{X} \cap M\right)^{\circ}=B_{X}^{\circ}+M^{\perp},(M+A)^{\circ}=M^{\perp} \cap A^{\circ}, T\left(B_{X}\right)^{\circ}=T^{\prime-1}\left(B_{X}^{\circ}\right)$ and $\left[T^{-1}\left(B_{Y}\right)\right]^{\circ}=T^{\prime}\left(B_{Y}^{\circ}\right)$ (where the circle denotes the polar of a set).

Proof. The proof of (i) is classical and for the proof of (ii) see [6].

Definition 1. Let $X, Y$ be normed spaces and $T: X \rightarrow Y$ a linear operator. We say that the operator $T$ is open if there exists $\varrho>0$ such that

$$
\varrho B_{Y} \cap R(T) \subset T\left(B_{X}\right) .
$$


Remark 2. If $X, Y$ are normed spaces and $T \in \mathcal{B}(X, Y)$ then $T$ is open if and only if the map $X \ni x \mapsto T x \in R(T)$ takes open sets in $X$ into open sets in $R(T)$.

Proposition 1. Let $X, Y$ be normed spaces, $T \in \mathcal{B}(X, Y)$ and $K \in$ $\mathcal{K}(X, Y)$. If $T$ is open and $\operatorname{codim} R(T)<\infty$, then $T+K$ is open.

Proof. First of all we show that the adjoint $T^{\prime} \in \mathcal{B}\left(Y^{\prime}, X^{\prime}\right)$ is open. Because $T$ is open, from Lemma 2 it follows that there exists $\varrho>0$ such that

$$
T\left(B_{X}\right)^{\circ} \subset\left[\varrho B_{Y} \cap R(T)\right]^{\circ}=R(T)^{\perp}+\frac{1}{\varrho} B_{Y}^{\circ} .
$$

Using again Lemma 2 and (6) we deduce that

$$
T^{\prime-1}\left(B_{X}^{\circ}\right) \subset N\left(T^{\prime}\right)+\frac{1}{\varrho} B_{Y}^{\circ} .
$$

Hence,

$$
\varrho B_{X}^{\circ} \cap R\left(T^{\prime}\right) \subset T^{\prime}\left(B_{Y}^{\circ}\right),
$$

that is, $T^{\prime}$ is open. Remark 2 implies that $R\left(T^{\prime}\right)$ is closed. From

$$
\operatorname{dim} N\left(T^{\prime}\right)=\operatorname{codim} \overline{R(T)} \leq \operatorname{codim} R(T)<\infty
$$

it follows that $T^{\prime}$ has an index. Applying [5, Corollary V.2.2] we find that $R\left(T^{\prime}+K^{\prime}\right)$ is closed, and because $T^{\prime}+K^{\prime}$ is continuous, it follows that $T^{\prime}+K^{\prime}: Y^{\prime} \rightarrow R\left(T^{\prime}+K^{\prime}\right)$ is open. This implies that there exists $\varrho>0$ such that

$$
\varrho B_{X}^{\circ} \cap R\left(T^{\prime}+K^{\prime}\right) \subset\left(T^{\prime}+K^{\prime}\right)\left(B_{Y}^{\circ}\right) .
$$

Using (7), Lemma 2 and the closedness of $R\left(T^{\prime}+K^{\prime}\right)$ we obtain

$$
\varrho\left[B_{X}+N(T+K)\right]^{\circ} \subset\left[(T+K)^{-1}\left(B_{Y}\right)\right]^{\circ} .
$$

Taking the polars of both sides in (8) and using the bipolar theorem it follows that

$$
\begin{aligned}
\varrho(T+K)^{-1}\left(B_{Y}\right) & =\varrho\left[(T+K)^{-1}\left(B_{Y}\right)\right]^{\circ \circ} \subset\left[B_{X}+N(T+K)\right]^{\circ \circ} \\
& =\overline{B_{X}+N(T+K)} \subset 2 B_{X}+N(T+K),
\end{aligned}
$$

which yields

$$
\varrho B_{Y} \cap R(T+K) \subset 2(T+K)\left(B_{X}\right),
$$

that is, $T+K$ is open.

REMARK 3. If $T \in \mathcal{B}(X, Y)$ is open then the proof above shows that $R\left(T^{\prime}\right)$ is closed. 
3. Proof of the main result. The product of two normed spaces $X_{1}$ and $X_{2}$ will be endowed with the norm

$$
\left\|\left(x_{1}, x_{2}\right)\right\|=\left(\left\|x_{1}\right\|^{2}+\left\|x_{2}\right\|^{2}\right)^{1 / 2}, \quad \forall\left(x_{1}, x_{2}\right) \in X_{1} \times X_{2} .
$$

Lemma 3. Let $X$ and $Y$ be normed spaces, $X_{0} \subset X$ a closed linear subspace and $Y_{0} \subset Y$ a linear subspace. Let $T_{0} \in \mathcal{B}(X, Y)$ and $K_{0} \in \mathcal{K}(X, Y)$ be such that $T_{0}\left(X_{0}\right) \subset Y_{0}$ and $K_{0}\left(X_{0}\right) \subset Y_{0}$. Suppose that $T_{0}$ is open, $K_{0} \mid X_{0}: X_{0} \rightarrow Y_{0}$ is compact, and $R\left(T_{0}\right)+Y_{0}, R\left(T_{0}+K_{0}\right)+Y_{0}$ are closed subspaces of $Y$. Let $T, K: X / X_{0} \rightarrow Y / Y_{0}$ induced by $T_{0}$ and $K_{0}$ respectively. If $T$ is Fredholm, then $T+K$ is Fredholm and $\operatorname{ind}(T)=\operatorname{ind}(T+K)$.

Proof. Associate to $T+K$, as in the case of $T$, the sequence of normed spaces

$$
0 \rightarrow X_{0} \stackrel{i_{T+K}}{\longrightarrow} X \times Y_{0} \stackrel{j_{T+K}}{\longrightarrow} Y \rightarrow 0
$$

where

$$
\begin{aligned}
i_{T+K}\left(x_{0}\right) & =\left(x_{0},\left(T_{0}+K_{0}\right) x_{0}\right), \quad \forall x_{0} \in X_{0}, \\
j_{T+K}\left(x, y_{0}\right) & =\left(T_{0}+K_{0}\right) x-y_{0}, \quad \forall x \in X, y_{0} \in Y_{0} .
\end{aligned}
$$

It is easy to check that (9) is a complex.

(a) Some properties of $i_{T}, j_{T}, i_{T+K}$ and $j_{T+K}$. It is clear that $i_{T} \in$ $\mathcal{B}\left(X_{0}, X \times Y_{0}\right)$ and $R\left(i_{T}\right)$ is closed. On the other hand,

$$
\left\|x_{0}\right\| \leq\left\|\left(x_{0}, T_{0} x_{0}\right)\right\|=\left\|i_{T}\left(x_{0}\right)\right\| \text {. }
$$

It follows that

$$
B_{X \times Y_{0}} \cap R\left(i_{T}\right) \subset i_{T}\left(B_{X_{0}}\right),
$$

hence $i_{T}$ is open. Clearly, $j_{T} \in \mathcal{B}\left(X \times Y_{0}, Y\right)$. Because $R\left(T_{0}\right)+Y_{0}$ is closed, the map $\beta$ from the proof of Lemma 1 is continuous. As $R\left(j_{T}\right)=N(\beta)$, it follows that $R\left(j_{T}\right)$ is closed. Consider the maps

$$
\begin{gathered}
s: X \times Y_{0} \rightarrow R\left(T_{0}\right) \times Y_{0}, \quad s\left(x, y_{0}\right)=\left(T_{0} x, y_{0}\right), \\
t: R\left(T_{0}\right) \times Y_{0} \rightarrow Y, \quad t\left(T_{0} x, y_{0}\right)=T_{0} x-y_{0} .
\end{gathered}
$$

Notice that $t$ is open. Because $T_{0}$ is open, it follows easily that $s$ is open. Hence, $j_{T}=t \circ s$ is open. On the other hand, $\operatorname{codim} R\left(T_{0}\right)=\operatorname{codim} R(T)<\infty$. Hence, Proposition 1 shows that $T_{0}+K_{0}$ is open. So, we can replace $T_{0}$ with $T_{0}+K_{0}$ to conclude that $i_{T+K}$ and $j_{T+K}$ have the same properties as $i_{T}$ and $j_{T}$.

(b) The adjoints of complexes (4) and (9). We consider the sequences

$$
\begin{gathered}
0 \rightarrow Y^{\prime} \stackrel{j_{T}^{\prime}}{\longrightarrow} X^{\prime} \times Y_{0}^{\prime} \stackrel{i_{T}^{\prime}}{\longrightarrow} X_{0}^{\prime} \rightarrow 0, \\
0 \rightarrow Y^{\prime} \stackrel{j_{T+K}^{\prime}}{\longrightarrow} X^{\prime} \times Y_{0}^{\prime} \stackrel{i_{T+K}^{\prime}}{\longrightarrow} X_{0}^{\prime} \rightarrow 0 .
\end{gathered}
$$

Using Lemma 2 and Remark 3 it follows that (10), (11) are complexes, (10) is Fredholm, and

$$
\operatorname{ind}(4)=\operatorname{ind}(10) \text {. }
$$


Moreover, (9) is Fredholm iff (11) is Fredholm, and in this case,

$$
\operatorname{ind}(9)=\operatorname{ind}(11) \text {. }
$$

We will use Theorem 2 to prove that (11) is Fredholm and ind(10) $=\operatorname{ind}(11)$. To do this, we write (11) as a compact perturbation of (10) as follows. Consider the compact operators

$$
\begin{gathered}
i: X_{0} \rightarrow X \times Y_{0}, \quad i\left(x_{0}\right)=\left(0, K_{0} x_{0}\right), \\
j: X \times Y_{0} \rightarrow Y, \quad j\left(x, y_{0}\right)=K_{0} x .
\end{gathered}
$$

Note that $i_{T+K}=i_{T}+i$ and $j_{T+K}=j_{T}+j$. Because $i$ and $j$ are compact it follows that $i_{T+K}^{\prime}-i_{T}^{\prime}$ and $j_{T+K}^{\prime}-j_{T}^{\prime}$ are compact. Applying Theorem 2 we deduce that (11) is Fredholm and

$$
\operatorname{ind}(10)=\operatorname{ind}(11) \text {. }
$$

(c) End of proof. Using Lemma 1 and (12)-(14) we find that

$$
\operatorname{ind}(T)=\operatorname{ind}(T+K) \text {. }
$$

Let $X$ be a normed space and $M:=\{x \in X:\|x\|=1\}$. Define

$$
l_{0}^{1}(M):=\{\lambda: M \rightarrow \mathbb{C}: \operatorname{supp} \lambda \text { is finite }\},
$$

where $\operatorname{supp} \lambda=\{x \in M: \lambda(x) \neq 0\}$. We endow the vector space $l_{0}^{1}(M)$ with the norm $\|\lambda\|=\sum_{x \in M}|\lambda(x)|$. Consider the linear operator

$$
S_{0}: l_{0}^{1}(M) \rightarrow X, \quad S_{0} \lambda=\sum_{x \in M} \lambda(x) x .
$$

Obviously, $S_{0} \in \mathcal{B}\left(l_{0}^{1}(M), X\right)$ and $S_{0}$ is surjective. Define the linear operator

$$
S: l_{0}^{1}(M) / N\left(S_{0}\right) \rightarrow X, \quad S\left(\lambda+N\left(S_{0}\right)\right)=S_{0} \lambda .
$$

Then $S$ is continuous and bijective.

Let $T \in \operatorname{LR}(X, Y)$ be a linear relation and $q_{T}: Y \rightarrow Y / T(0)$ be the canonical surjection. Associate to $T$, as in [3, Section I.6], the linear transformation

$$
q_{T} T: X \rightarrow Y / T(0), \quad\left(q_{T} T\right)(x)=y+T(0),
$$

where $y \in T x$ is arbitrarily chosen. We see that $R_{0}\left(q_{T} T\right)=R(T)$ and (15) $T$ is Fredholm iff $q_{T} T$ is Fredholm, and $\operatorname{ind}(T)=\operatorname{ind}\left(q_{T} T\right)$.

Proof of Theorem 1. We denote $N\left(S_{0}\right)$ by $X_{0}$ and $T(0)$ by $Y_{0}$. Consider the linear relation $T S \in \operatorname{LR}\left(l_{0}^{1}(M) / X_{0}, Y\right)$. Because $S$ is bijective, we have $T(0)=(T S)(0)$, and hence $(15)$ shows that $\operatorname{ind}(T)=\operatorname{ind}\left(q_{T} T\right)=$ $\operatorname{ind}\left(q_{T}(T S)\right)=\operatorname{ind}(Q)$, where $Q=q_{T} T S$. Thus

$$
\operatorname{ind}(Q)=\operatorname{ind}(T) \text {. }
$$


Consider

$$
\begin{array}{ll}
q_{1}: G_{0}(Q) \rightarrow l_{0}^{1}(M), & q_{1}(\lambda, y)=\lambda, \\
q_{2}: G_{0}(Q) \rightarrow Y, & q_{2}(\lambda, y)=y .
\end{array}
$$

Clearly $q_{1}, q_{2}$ are continuous and $q_{2}$ is open. On the other hand, the map $q_{1}$ takes open sets in $G_{0}(Q)$ to open sets in $R\left(q_{1}\right)$, hence

$$
\gamma\left(q_{1}\right):=\sup \left\{\delta>0: \delta d\left(\xi, N\left(q_{1}\right)\right) \leq\left\|q_{1} \xi\right\|, \forall \xi \in G_{0}(Q)\right\}>0 .
$$

Let

$$
Q_{1}: G_{0}(Q) /\left(X_{0} \times Y_{0}\right) \rightarrow l_{0}^{1}(M) / X_{0}, \quad Q_{2}: G_{0}(Q) /\left(X_{0} \times Y_{0}\right) \rightarrow Y / Y_{0}
$$

be induced by $q_{1}$ and $q_{2}$. It follows easily that $Q_{1}$ is bijective and $Q=Q_{2} \circ$ $Q_{1}^{-1}$. For $x \in M$, let $e_{x} \in l_{0}^{1}(M)$ be such that $e_{x}(x)=1$ and $\operatorname{supp} e_{x}=\{x\}$. Using the fact that $q_{1}$ is surjective and $\gamma\left(q_{1}\right)>0$ we deduce that for all $x \in M$ there exists $\xi_{x}=\left(e_{x}, y_{x}\right) \in G_{0}(Q)$ such that $\left\|\xi_{x}\right\| \leq r$, where $r>\gamma\left(q_{1}\right)^{-1}$. Define the linear operator

$$
q_{0}: l_{0}^{1}(M) \rightarrow G_{0}(R), \quad q_{0}(\lambda)=\sum_{x \in M} \lambda(x) \xi_{x}=\left(\lambda, \sum_{x \in M} \lambda(x) y_{x}\right) .
$$

From the choice of $\xi_{x}$ it follows that $q_{0}$ is continuous and a simple computation shows that $q_{0}$ is open. Let

$$
T_{0}: l_{0}^{1}(M) \rightarrow Y, \quad T_{0}=q_{2} \circ q_{0} .
$$

The linear operator $T_{0}$ is continuous open and because $q_{2}$ induces $Q_{2}$ and $q_{0}$ induces $Q_{1}^{-1}$, we deduce that $T_{0}$ induces $Q$. Using

$$
R(T)=R(T S)=R_{0}(Q)=R\left(T_{0}\right)+Y_{0}
$$

and the closedness of $R(T)$ it follows that $R\left(T_{0}\right)+Y_{0}$ is closed.

Let $\tau: l_{0}^{1}(M) \rightarrow l_{0}^{1}(M) / X_{0}$ be the canonical surjection and

$$
K_{0}: l_{0}^{1}(M) \rightarrow Y, \quad K_{0}=K S \tau .
$$

Note that $K_{0}$ is compact and $K_{0} \mid X_{0}$ is identically zero. Let

$$
\widetilde{Q}=q_{(T+K) S}(T+K) S=q_{T}(T+K) S .
$$

We have

$$
\begin{aligned}
\widetilde{Q}\left(\lambda+X_{0}\right) & =\left[q_{T}(T+K) S\right]\left(\lambda+X_{0}\right)=Q\left(\lambda+X_{0}\right)+q_{T} K S \tau(\lambda) \\
& =T_{0}(\lambda)+Y_{0}+q_{T} K_{0}(\lambda)=T_{0}(\lambda)+K_{0}(\lambda)+Y_{0},
\end{aligned}
$$

that is, $\widetilde{Q}$ is induced by $T_{0}+K_{0}$. From

$$
R(T+K)=R((T+K) S)=R_{0}(\widetilde{Q})=R\left(T_{0}+K_{0}\right)+Y_{0}
$$

and the closedness of $R(T+K)$ it follows that $R\left(T_{0}+K_{0}\right)+Y_{0}$ is closed.

We are now in a position to apply Lemma 3 to the operators $T_{0}$ and $K_{0}$. It follows that $\widetilde{Q}$ is Fredholm and

$$
\operatorname{ind}(Q)=\operatorname{ind}(\widetilde{Q})
$$


On the other hand, using (15) we see that $(T+K) S$ is Fredholm iff $\widetilde{Q}$ is Fredholm, and

$$
\operatorname{ind}((T+K) S)=\operatorname{ind}(\widetilde{Q}) .
$$

From the bijectivity of $S$ and (16)-(18) the conclusion of the theorem follows.

REMARK 4. Let $X$ be a normed space. If $K \in \mathcal{K}(X)$, then ind $(I-K)=0$. In particular $I-K$ is injective iff $I-K$ is surjective.

Remark 5. Let $X, Y$ be normed spaces, $T: X \rightarrow Y$ a linear bijection and $K \in \mathcal{K}(X, Y)$. Suppose that the equation

$$
T x+K x=0
$$

has at least one nontrivial solution. Then there exists $y \in Y$ such that the equation

$$
T x+K x=y
$$

has no solution.

\section{References}

[1] C.-G. Ambrozie, The Euler characteristic is stable under compact perturbations, Proc. Amer. Math. Soc. 127 (1996), 2041-2050.

[2] C.-G. Ambrozie and F.-H. Vasilescu, Banach Space Complexes, Math. Appl. 334, Kluwer, Dordrecht, 1995.

[3] R. W. Cross, Multivalued Linear Operators, Dekker, New York, 1998.

[4] D. Gheorghe, Stability of the index of linear operators between quotient normed spaces, preprint, 2006.

[5] S. Goldberg, Unbounded Linear Operators. Theory and Applications, McGraw-Hill, New York, 1966.

[6] R. Menniken, Perturbations of semi-Fredholm operators in locally convex spaces, in: Functional Analysis, Holomorphy, and Approximation Theory (Rio de Janeiro, 1979), Lecture Notes in Pure and Appl. Math. 83, Dekker, New York, 1983, 233-304.

[7] F.-H. Vasilescu, Stability of the index of a complex of Banach spaces, J. Operator Theory 2 (1979), 247-275.

[8] H. Zhang, Fredholm theory for morphisms in quotient Banach spaces, Rev. Roumaine Math. Pures Appl. 34 (1989), 309-316.

Facultatea de matematica-informatica Université des Sciences et Technologies de Lille Universitatea din Pitesti 59655 Villeneuve d'Ascq Cedex, France

Str. Targul din Vale, nr. 1

110040 Pitesti, Arges, Romania

E-mail: gheorghedana@yahoo.com

Received June 19, 2006

Revised version February 13, 2007 